\title{
Particularités des matériels militaires de radioprotection*
}

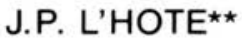 \\ (Manuscrit reçu le 19 avril 1983) \\ RÉSUMÉ
}

\begin{abstract}
Les matériels de radioprotection développés pour les besoins de la défense nucléaire sont particuliers par leur fonction et leur mode d'utilisation. Par leur fonction, ils sont destinés à être utilisés en cas d'explosion nucléaire, d'où un certain nombre de caractéristiques qui les rendent spécifiques. Par leur mode d'utilisation, ils sont destinés à être mis en œuvre sur le champ de bataille, d'où des spécifications d'environnement contraignantes. En conclusion, un aperçu des tendances technologiques actuelles pour réaliser les nouveaux matériels est présenté.
\end{abstract}

\section{ABSTRACT}

The health physics equipments that have been developed for nuclear defense, are particular by their function and by their operating use. By their function, they are assigned to be used in case of a nuclear burst. Consequently, they have a number of specific characteristics. By their operating use, they are assigned to operate on the battle field. Consequently, they have constricting environmental specifications. In conclusion, a survey of the present technological trends in the realization of new devices is presented.

\section{INTRODUCTION}

Les matériels de radioprotection développés pour les besoins de la défense nucléaire sont particuliers. Ils le sont par leur fonction. En effet, ils sont prévus pour être utilisés en cas d'explosion nucléaire, et, de ce fait, ont des spécifications techniques particulières. Ils sont également particuliers par leur emploi. Ils sont prévus pour être utilisés dans le cadre du champ de bataille et, par suite, doivent satisfaire à toutes les exigences des matériels militaires.

* Exposé présenté lors de la Journée d'études organisée conjointement par l'Etablissement technique central de l'armement et la Société française de radioprotection à Arcueil, le 3 juin 1982.

** Etablissement technique central de l'armement, Centre d'études du Bouchet, BP 3, 91170 Vert-le-Petit. 


\section{FONCTION DES MATERIELS}

\section{II.1. Rappels sur les effets nucléaires d'une explosion}

Les effets dus aux rayonnements nucléaires issus de l'explosion d'une arme nucléaire sont constitués du rayonnement initial et du rayonnement résiduel.

L'effet nucléaire initial se traduit par l'émission de rayonnement gamma et de neutrons. Les fluences, durées d'émission, doses et spectres d'énergie dépendent du type d'arme, de sa puissance et de l'endroit où l'on se trouve par rapport au point de l'explosion.

L'effet nucléaire résiduel est dû d'une part à la radioactivité induite par les neutrons au voisinage de l'explosion et d'autre part aux retombées radioactives.

Les retombées sont constituées de poussières radioactives provenant des débris de l'arme et des matériaux du sol aspirés par l'explosion. La radioactivité est due principalement à des produits de fission émetteurs $\beta$ et $\gamma$. Le spectre énergétique du rayonnement gamma a pour énergie moyenne 0,7 MeV. Le débit de dose $A$ dû aux retombées sur le terrain décroît au cours du temps selon la relation approchée suivante :

$$
\frac{A}{A_{1}}=\left(\frac{t}{t_{1}}\right)^{-1,2}
$$

où $t$ est le temps écoulé après l'explosion et $A_{1}$ le débit de dose au temps $t_{1}$ après l'explosion ; par commodité $t_{1}$ est souvent pris égal à $1 \mathrm{~h}$ après l'explosion.

La répartition des retombées sur le terrain dépend de nombreux facteurs, à savoir la puissance de l'arme, l'altitude de l'explosion, le temps après l'explosion, les conditions climatiques et le relief. Dans certaines configurations, les distances peuvent être considérables.

\section{II.2. Rôle des matériels}

L'explosion nucléaire engendre, dans le domaine nucléaire, deux types de nuisances : un risque d'irradiation externe à distance, un risque de contamination.

Ces deux types de risques rendent nécessaires :

- Putilisation de dosimètres permettant le suivi des doses "neutron et gamma" recues par le personnel exposé et dues au rayonnement instantané et le suivi des doses gamma dues aux retombées; le même appareil peut éventuellement servir dans les deux cas ;

- la mesure des débits de doses ou l'obtention d'alarmes à l'aide de débitmètres portatifs ou portables ;

- la détermination des zones contaminées et leur délimitation avec des débitmètres portatifs ou installés sur véhicule, le marquage de ces zones ;

- la protection du personnel ou du matériel contre la contamination, d'où des équipements vestimentaires adaptés, des systèmes de nettoyage, des appareils de mesure de la contamination. 
On trouve tout ce qu'il est nécessaire de prévoir du point de vue nucléaire, dans le domaine civil. Les spécifications techniques du matériel pour la défense nucléaire sont, cependant, particulières. Elles ne correspondent pas à celles des matériels civils. Nous nous limitons dans ce qui suit aux radiamètres.

\section{II.3. Caractéristiques fonctionnelles des radiamètres}

Une des caractéristiques originales de ce type de matériel est la gamme de mesure :

- En dosimétrie, elle va de 1 cGy à 1000 cGy. La valeur inférieure est élevée si l'on se réfère à ce qui est utilisé couramment. II ne faut pas perdre de vue que la dosimétrie sur le champ de bataille a pour but de permettre de contrôler la capacité opérationnelle des personnels. Pour les détecteurs "neutron et gamma" ceci est la gamme de mesure pour chaque type de rayonnement ou pour la somme des deux contributions.

- En débitmétrie, la gamme de mesure normale va de $0,1 \mathrm{cGy} / \mathrm{h}$ à $500 \mathrm{cGy} / \mathrm{h}$. De nombreux appareils ont une extension vers $1000 \mathrm{cGy} / \mathrm{h}$. Certains comme le DOM 410 (fig. 1) ont également des applications dans le domaine civil et leur gamme débute à $10^{-3} \mathrm{cGy} / \mathrm{h}$. Les niveaux d'alarme sont, en général, dans la gamme de mesure du débit. Ainsi, le DUK DUR $\mathbf{4 4 0}$ (fig. 2), appareil équipant des véhicules, déclenche l'alarme à partir d'un débit de $1 \mathrm{cGy} / \mathrm{h}$.

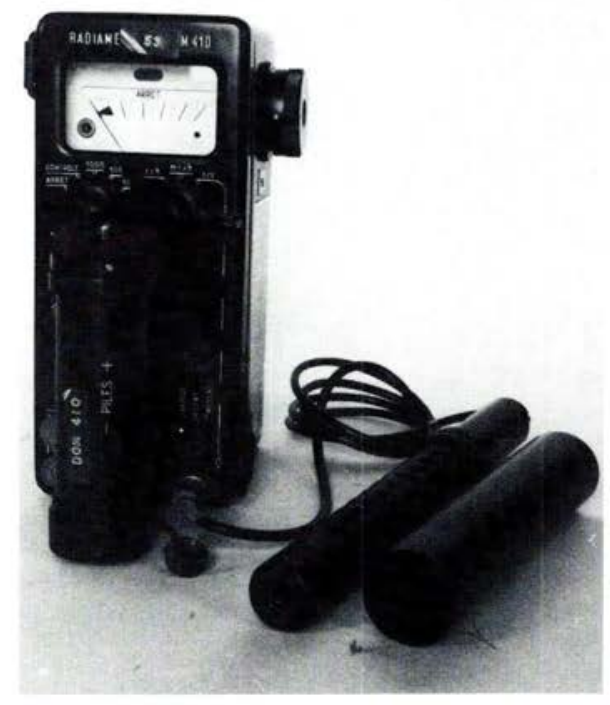

Fig.1. - Débitmètre DOM 410 - stade série - fabriqué par la société Saphymo-Stel. 


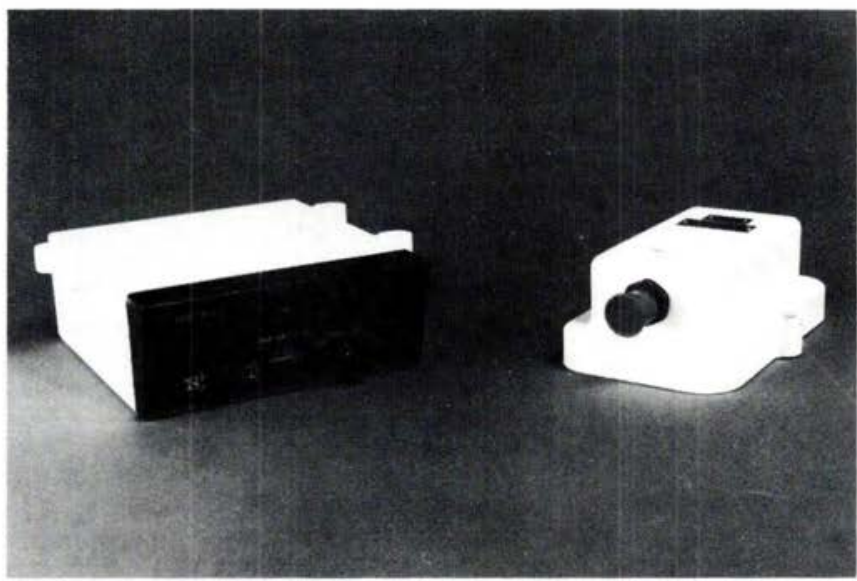

Fig. 2. - Débitmètre - dosimètre DUK DUR 440 stade présérie - fabriqué par la Manufacture d'Armes de Saint-Etienne.

- En mesure de contamination, la tendance actuelle est de pouvoir effectuer les mesures de contamination $\gamma$ et $\beta$ en utilisant une sonde dont la sensibilité est limitée intentionnellement. Les informations fournies sont exprimées en unités arbitraires. Le niveau de sensibilité est équivalent en gamma à un débitmètre dont la gamme irait de $10^{-3} \mathrm{cGy} / \mathrm{h}$ à $1 \mathrm{cGy} / \mathrm{h}$ pour le césium 137.

Une autre spécification est très liée à ce que l'on désire mesurer ; il s'agit de la gamme d'énergie du rayonnement pour laquelle l'appareil doit répondre de façon uniforme :

- Pour le rayonnement instantané, des prototypes sont en cours d'étude. Le problème est très complexe pour les neutrons, pour lesquels il n'existe pas de détecteur universel, c'est-à-dire ayant une réponse indépendante de l'énergie des neutrons. Les spécifications admettent une précision de $\pm 40 \%$.

- Pour le rayonnement de retombées, les appareils doivent répondre pour des énergies de rayonnement allant de $80 \mathrm{keV}$ à $3 \mathrm{MeV}$ (souhaitable de $50 \mathrm{keV}$ à $3 \mathrm{MeV}$ ) avec une précision de $\pm 20 \%$.

- Pour le rayonnement $\beta$, le seuil de sensibilité résulte d'un compromis entre la solidité du matériel (il vaut mieux éviter, par exemple, les tubes à fenêtre) et la valeur minimale d'énergie intéressante à mesurer. Le seuil choisi est de $250 \mathrm{keV}$.

Parmi les caractéristiques nucléaires spécifiques figure également la saturation. Un débitmètre doit, en effet, pouvoir supporter pendant quelques minutes un débit de $10 \mathrm{~Gy} / \mathrm{h}$ sans baisse de son indication ni mauvais fonctionnement. 


\section{EMPLOI DES MATÉRIELS}

Les matériels de radioprotection développés pour la défense nucléaire sont destinés à être utilisés sur le champ de bataille. Ils doivent permettre l'obtention de l'information avec la précision désirée (souvent de $\pm 20 \%$ ) et avec les caractéristiques d'emploi suivantes :

\section{III.1. La simplicité}

La simplicité se manifeste au niveau de la conception de Pappareil par la simplicité de mise en œuvre, la clarté des indications, l'existence d'automatismes. Elle se manifeste également par des exigences d'encombrement, d'autonomie, par du matériel de maintenance adapté. La simplicité pour P'exploitation des résultats peut entraîner des fonctions supplémentaires dans l'appareil. Ainsi le JUK 450 (fig. 3 ) qui est un dé bitmètre monté sur aéronef fournit directement le débit de dose à $1 \mathrm{~m}$ du sol et ramène à $1 \mathrm{~h}$ après l'explosion à partir du débit mesuré à l'altitude de vol et à l'instant $t$ après l'explosion. Ceci permet d'avoir une information exploitable plus facilement par le personnel au sol.

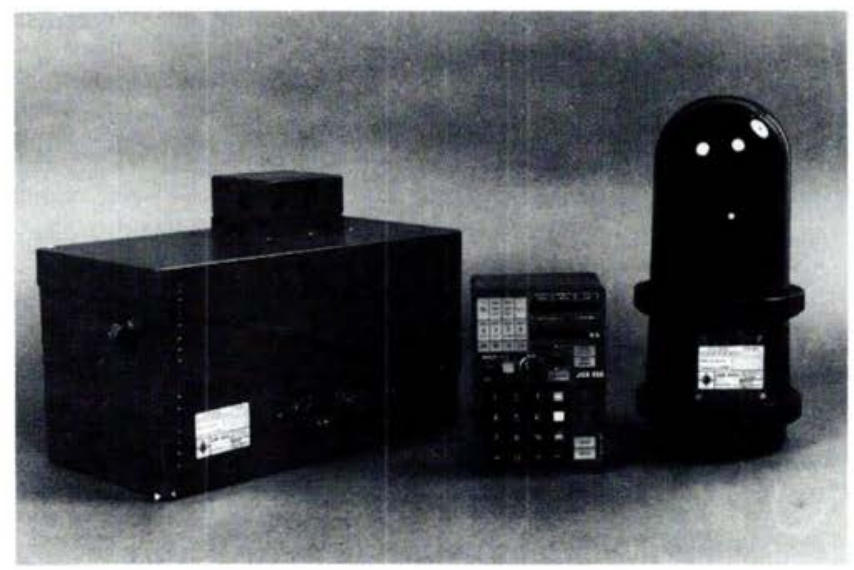

Fig.3.- Débitmètre JUK 450 - stade série fabriqué par la société BEFIC.

L'appareil sera d'autant plus simple à manipuler que l'opérateur y aura été préparé. Dans ce but, sont conçus en même temps que les matériels, des appareils permettant l'instruction des personnels. Cette instruction est individuelle et, dans ce cas, l'appareil d'instruction est soit un accessoire associé au vrai radiamètre, soit un appareil spécifique, réplique exacte du radiamètre. Son but est d'entraîner le personnel à mettre en œuvre le radiamètre, à lire ses indications sans devoir utiliser 
une source radioactive. L'instruction peut être également collective (lors de manœuvres, par exemple). Dans ce cas, un champ radioélectrique est utilisé pour simuler le rayonnement sur la zone de retombées. Le pseudo radiamètre de même aspect extérieur que le vrai est un mesureur de champ.

\section{III.2. La rapidité}

La notion de rapidité est liée au temps de réponse de l'appareil et au temps de mise en œuvre. La nécessité de pouvoir rapidement délimiter des zones implique la conception d'appareils utilisés sur véhicule comme le JUK 450 déjà cité.

\section{III.3. La fiabilité}

La fiabilité est liée aux caractéristiques électriques classiques et aux conditions d'environnement auxquelles est soumis le matériel dans son utilisation. Ces conditions d'environnement correspondent à un certain nombre d'actions résultant de l'emploi du matériel.

Ce sont des conditions mécaniques liées à P'utilisation et au transport. Par exemple, le matériel doit être transporté par tout moyen terrestre, maritime et aérien (jusqu'à $15000 \mathrm{~m}$ ), résister aux chutes, chocs, vibrations résultant de l'utilisation et du transport, être étanche à l'immersion, fonctionner entre 0 et $3000 \mathrm{~m}$.

Ce sont des conditions liées à l'environnement climatique, à savoir pouvoir fonctionner à des températures comprises par exemple entre $-25^{\circ} \mathrm{C}$ et $+60^{\circ} \mathrm{C}$, fonctionner à $+55^{\circ} \mathrm{C}$ sous $93 \%$ d'humidité, résister à l'air salin et aux moisissures, pouvoir être stocké entre $-50^{\circ} \mathrm{C}$ et $+70^{\circ} \mathrm{C}$, supporter les surpressions.

Ce sont également des conditions liées à l'environnement nucléaire, à savoir la possibilité de décontaminer le matériel, la résistance aux effets nucléaires, électromagnétiques et thermiques des armes. En outre, le matériel ne doit pas perturber ni être perturbé par les environnements radioélectriques.

Toutes ces exigences se traduisent par un nombre d'essais très important lors de la réception des matériels.

\section{EFFETS DES SPECCIFICATIONS SUR LES CHOIX TECHNOLOGIQUES - TENDANCES ACTUELLES}

Dans les cahiers de charges, la tendance actuelle est d'augmenter le nombre de fonctions et de diminuer les dimensions des appareils, les caractéristiques d'environnement étant relativement stables.

Du point de vue technologique, les indicateurs à aiguilles sont de plus en plus remplacés par des afficheurs, le choix du type (cristaux li- 
quides ou diodes électroluminescentes) étant lié à l'utilisation. Les différentes fonctions sont gérées à l'aide de circuits électroniques utilisant des microprocesseurs. Ceci permet de diminuer notablement le nombre d'opérations que doit effectuer le manipulateur.

Le seul point noir reste encore la consommation puisqu'actuellement le volume des piles est souvent le même que celui de l'électronique.

Le prototype DOM DOR 460 (fig. 4) débitmètre dosimètre destiné à remplacer le DOM 410 (fig. 1) constitue, en quelque sorte, une synthèse de toutes ces innovations technologiques.

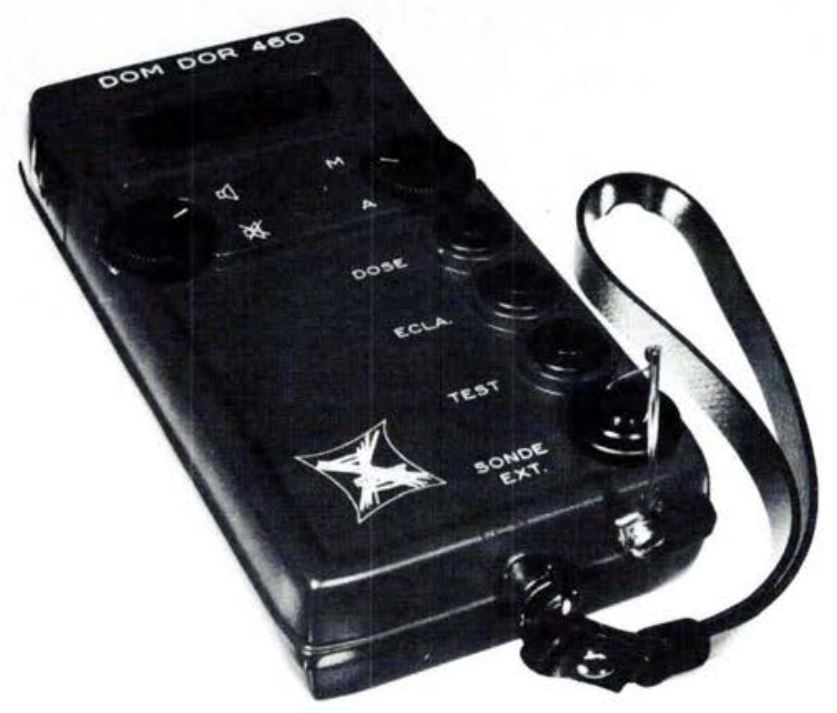

Fig. 4.- Débitmètre- dosimètre DOM DOR 460 - stade prototypefabriqué par la société Nardeux. 